\title{
Evaluation of the hepatoprotective effect of Yigan mingmu oral liquid against acute alcohol-induced liver injury in rats
}

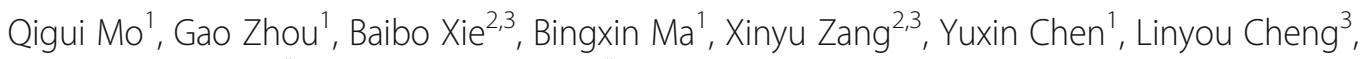
James Hua Zhou ${ }^{2,3^{*}}$ and Youwei Wang ${ }^{1,4^{*}}$ (D)

\begin{abstract}
Background: Yigan mingmu oral liquid (YGMM) is a herbal medicine based on a famous Chinese herbal formula that has been used for sore eyes for more than 400 years. Eye health is closely associated with the liver based on TCM. This study aimed to investigate the hepatoprotective effect of YGMM against acute liver injury induced by alcohol in rats.

Methods: Experimental rats were administered with silymarin and YGMM through the gastric gavage during the entire experiment. Starting from the 11th day, the rats were administered orally with $14 \mathrm{ml} / \mathrm{kg}$ Red Star Erguotou Liquor, a popular brand, at $4 \mathrm{~h}$ after the dose of silymarin $(100 \mathrm{mg} / \mathrm{kg})$ and $Y G M M(1,2.5$ and $5 \mathrm{ml} / \mathrm{kg}$ in low, middle and high dosage group, respectively) once a day for 4 weeks except for the rats in the normal group. Biochemical parameters, including ALT, AST, TB, TG, T-SOD, GSH, and MDA were detected to evaluate the protective effect of YGMM. Pathological changes were observed through histopathological examination.
\end{abstract}

Results: Treatment with YGMM exhibited a significant protective effect by reversing the biochemical parameters (ALT, AST, TB, TG, and GSH) and histopathological changes. Histopathological examination by Oil Red O Staining Solution showed that lipid droplets were significantly reduced in the silymarin and YGMM groups $(p<0.001)$ when compared to alcohol group.

Conclusions: YGMM exhibits a significant hepatoprotective activity against acute liver injury induced by alcohol in rats.

Keywords: Yigan mingmu oral liquid, Hepatoprotective, Alcohol, Alcoholic liver disease, Oxidative stress

\section{Background}

Alcoholic liver disease (ALD) has recently become an important liver disease due to the increasing levels of alcohol consumption in the world [1]. The severity of this disease increases in the time and dose dependent manner with alcohol consumption and ranges initially from steatosis and steatohepatitis to fibrosis and cirrhosis [24]. However, the exact pathogenic mechanisms of ALD remain unclear. Previous studies have suggested that mitochondrial damage, generation of free radicals, and

\footnotetext{
*Correspondence: drjge@163.com; wyw@whu.edu.cn

2Beijing Hebabiz Biotechnology Co. Ltd, Beijing 102206, People's Republic of China

'Institute of TCM and Natural Products, School of Pharmaceutical Sciences, Wuhan University, Wuhan 430071, People's Republic of China Full list of author information is available at the end of the article
}

oxidative stress are important pathogenic events in the development of ALD [5, 6]. Supportive care and abstinence from alcohol are the only effective treatment methods. Moreover, some synthetic drugs, including bicyclol, tiopronin, and bifendate, have been used to alleviate the symptoms of ALD [7, 8]. Nevertheless, the definite and practical treatment strategies for ALD are still ambiguous. Developing traditional Chinese formulas and natural products with hepatoprotective effects has recently drawn attention $[9,10]$. Given their multitargeted and less toxic features, many herbal medicines have been investigated for ALD treatment [11].

Yigan mingmu oral liquid (YGMM) is a herbal medicine based on a famous Chinese herbal formula composed of 12 crude herbs: Rehmanniae Radix Preparata (Shudihuang),

(c) The Author(s). 2020 Open Access This article is distributed under the terms of the Creative Commons Attribution 4.0 International License (http://creativecommons.org/licenses/by/4.0/), which permits unrestricted use, distribution, and 
Angelicae Sinensis Radix (Danggui), Lycii Fructus (Gouqizi), Paeoniae Radix Alba (Baishao), Ophiopogonis Radix (Maidong), Chrysanthemi Flos (Juhua), Anemones Altaicae Rhizoma (Jiujiechangpu), Polygonati Odorati Rhizoma (Yuzhu), Chuanxiong Rhizoma (Chuanxiong), Citri Reticulatae Pericarpium (Chenpi), Cassiae Semen (Juemingzi), and Bupleuri Radix (Chaihu). This formula was approved by the CFDA as a therapeutic drug for sore eyes, lumbar debility, dizziness, hypomnesis, and body fatigue.

YGMM is based on Siwu decoction, an ancient formula first recorded in the secret formulary for traumatology and fracture taught by immortal in the Tang Dynasty and was used to treat trauma and extravasated blood. Siwu decoction was also found in some famous ancient medical books as treatment for obstetrical and gynecological diseases over the last hundred years. These books chronologically included Prescriptions of Peaceful Benevolent Dispensary in the Song Dynasty, Medical Formulae Investigations by Wu Kun in the Ming Dynasty, and Detailed Outline for Benefiting Female by Wu Zhiwang in the Ming Dynasty. Siwu decoction was initially composed of four Chinese herbals, namely, Angelicae Sinensis Radix, Chuanxiong Rhizoma, Paeoniae Radix Alba, and Rehmanniae Radix Preparata. In addition to the above herbals, Notopterygii Rhizoma et Radix, Saposhnikoviae Radix, and Angelicae Dahuricae Radix were incorporated in another famous formula named as Angelicae Sinensis Tonic Decoction, which was recorded in Shen Shi Yao Han by Fu Renyu in the Late Ming Dynasty and is usually used for sore eyes. Through incessant development and optimization, this formula was popularized in the Qing Dynasty and is still useful today. According to TCM, eye health is closely associated with the liver. Huang Di Nei Jing, one of the most famous ancient medical books in China, states that liver-qi is connected with the eyes; hence, the eyes can distinguish five colors when the liver-qi is unobstructed.

Pharmacological studies have showed that oxidative stress is an important pathogenesis for ALD [12-15]. Most of herbs in YGMM possess significant antioxidant activity [16-21], and some of them have been used for some diseases because of this property [22, 23]. More specifically, ferulic acid isolated from Angelicae Sinensis Radix and Chuanxiong Rhizoma possesses antioxidant activity and protection against alcohol-induced liver injury [24, 25]. Rehmanniae Radix Preparata, Lycii Fructus, and Paeoniae Radix Alba show antioxidant or anti-inflammatory activity [26-28]. Chrysanthemi Flos and Bupleuri Radix can also prevent liver injury [29, 30]. Besides, fat accumulation mainly results from lipid metabolic disorder, and is commonly observed in human and animal with ALD [31-33]. Bupleuri Radix and Polygonati Odorati Rhizoma could ameliorate fat metabolic disorder [34, 35], Ophiopogon japonicus and Paeonia moutan could suppress the hepatic lipid accumulation [36, 37]. Based on the above findings, we speculatedconjecture that YGMM could has hepatoprotective effects on the alcohol-induced liver injury.

In this study, the protective effects of YGMM on acute alcohol-induced liver injury in rats were investigated. The characteristics of liver injury were estimated by AST, ALT, and histopathological changes. In addition, T-SOD, GSH, MDA, TB, and TG levels in the hepatic tissues were detected to identify the possible mechanisms.

\section{Methods \\ YGMM}

YGMM samples were provided by Hebabiz Pharmaceutical (Guangxi, China). In brief, six TCM herbs, including Angelicae Sinensis Radix, Chrysanthemi Flos, Anemones Altaicae Rhizoma, Chuanxiong Rhizoma, Citri Reticulatae Pericarpium, and Bupleuri Radix, were crushed, from which the volatile oil was extracted by water distillation, meanwhile the filtrateI and residues were collected for further use. The residues and the rest six TCM herbs were decocted with water, and the decoction was filtered to obtain filtrate II. FiltrateI was combined with filtrateII, then concentrated into an ointment. The ointment was redissolved with ethanol, then the supernatant was concentrated after evaporating ethanol into a recovery tank. The concentrated supernatant was mixed with appropriate amount of water, honey, ethyl paraben and the collected volatile oil to produce YGMM.

\section{Animals}

Male Sprague-Dawley rats $(200 \pm 20 \mathrm{~g})$ were bought from the Center for Animal Experiment of Wuhan University, Wuhan, China. These experimental rats were allowed to adapt to feeding conditions for 1 week prior to the experiments. The feeding conditions were maintained at $25 \pm 2{ }^{\circ} \mathrm{C}, 40-70 \%$ relative humidity, and $12 \mathrm{~h}$ dark/light cycle. The rats had free access to standard rodent pellet diet and water during the assay period. The experimental protocol was approved by the Animal Ethics Committee of Wuhan University, Wuhan, China. Animal assays were conducted according to the guidelines of the Committee for the Purpose of Control and Supervision of Experiments on Animals.

\section{Drug administration}

After 1 week adaptation, the rats were assigned to the following six groups (six animals each) based on their body weight (BW): normal control, alcohol group, Silymarin group (as the positive control), and YGMM groups. According to preliminary experiments, the following daily doses were adopted for each treated group: the low, medium, and high YGMM doses were $1,2.5$, and $5 \mathrm{ml} / \mathrm{kg}$ BW, respectively, whereas silymarin dose was $100 \mathrm{mg} / \mathrm{kg}$ BW. The experimental process was showed in Fig. 1. In the beginning, the rats in the YGMM group were given a 


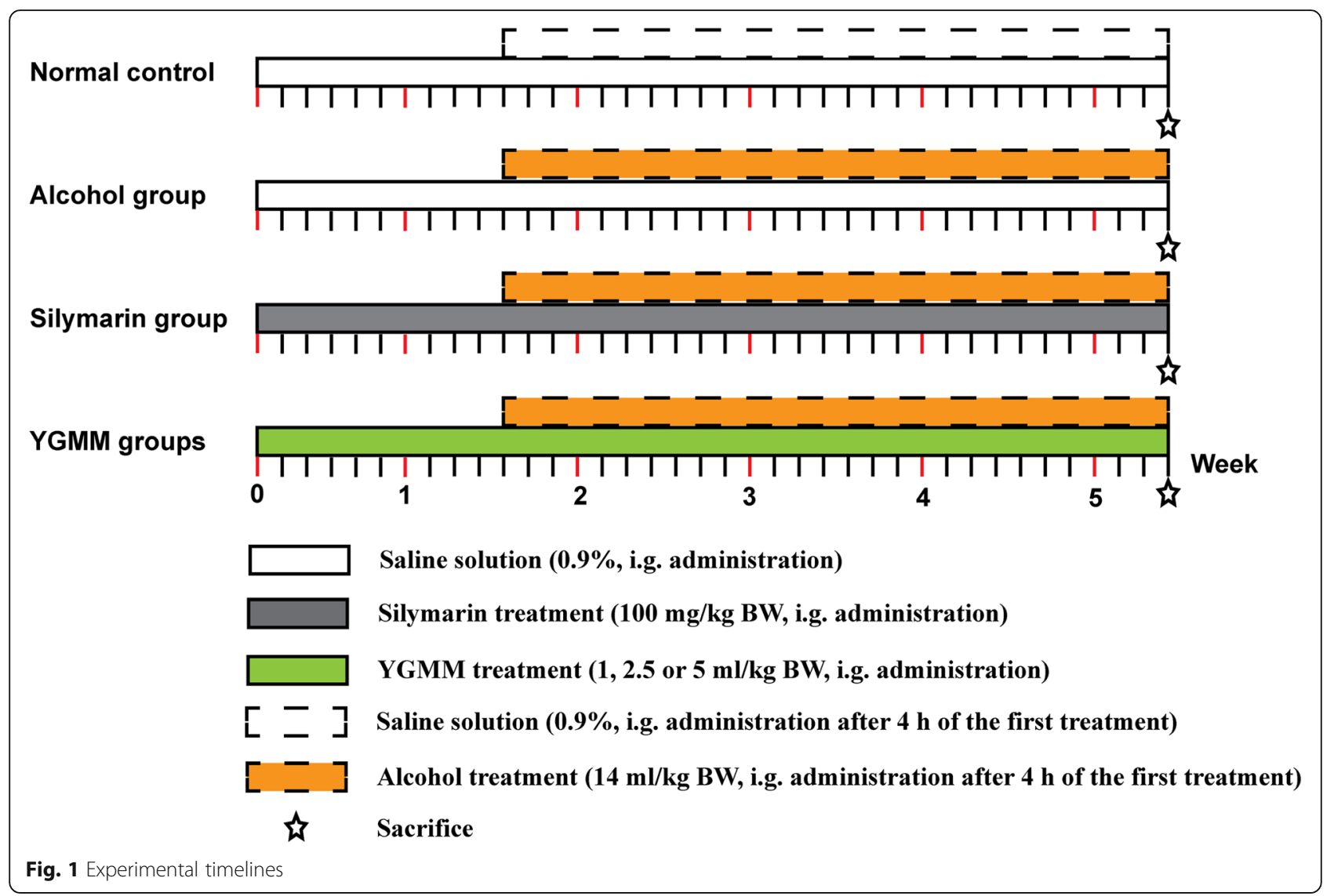

gavage of YGMM in three different doses, whereas those in the silymarin group were simultaneously fed with silymarin (Madaus GmbH, Cologne, Germany) through gastric gavage. The rats in the normal control and alcohol groups were given equal volumes of physiological saline. At the 11th day, after $4 \mathrm{~h}$ of treatment as mentioned above, all groups (except for the control group) were given a gavage of $14 \mathrm{ml} / \mathrm{kg}$ BW of Red Star Erguotou Liquor (Beijing Red Star, Beijing, China) once a day for 4 weeks. The liquor contains 56\% alcohol, so the dose approximately equals to $7.84 \mathrm{~g}$ alcohol per kilogram body weight. After the last treatment (day 38), the rats were fasted for $4 \mathrm{~h}$ and were executed by the cervical dislocation method. Blood samples were collected immediately and allowed to clot. The serums were obtained through centrifugation at $3000 \mathrm{rpm}$ for $8 \mathrm{~min}$ at $4{ }^{\circ} \mathrm{C}$ and were placed in ultra-lowtemperature freezer until the assay of AST, ALT, TB, and TG. The liver and kidney were excised, cleared of blood with ice-cold saline, and stored in a refrigerator immediately until the assay of T-SOD, GSH, and MDA.

\section{Biochemical assay}

AST, ALT activities and TB (Nanjing Jiancheng, Nanjing, China), TG (Shanghai Mind, Shanghai, China) levels were detected using the assay kits guided by the corresponding protocols.

Liver and kidney homogenates $(10 \%$, w/v) were prepared by using a high-speed dispersator (Ningbo Scientz, China) with ice-cold physiological saline. The clear supernatant liquid was obtained through centrifugation at $5000 \mathrm{rpm}$ for $5 \mathrm{~min}$ at $4{ }^{\circ} \mathrm{C}$ and used for T-SOD, GSH, and MDA assays (Nanjing Jiancheng, Nanjing, China). The assays were conducted using the kits according to the protocols of the manufacturer. Total protein concentration in the supernatant was detected according to Coomassie Brilliant Blue method.

\section{Histopathological studies}

Hematoxylin-eosin (H\&E) staining was performed first. The liver tissue was fixed with $10 \%$ formalin at normal temperature, and was subsequently embedded in paraffin after 1 day. The tissues were cut into $5 \mu$ m-thick slices and then stained with H\&E. Pathological section was examined in the blinding way under a light microscope.

Oil Red O staining was performed to observe the lipid droplet morphology as described in a previous study [38]. Quantitative analysis of the hepatic lipid content was conducted using Image-Pro Plus (Version 6.0). 
Table 1 Effect of YGMM on weight and organ index in control and alcohol-treated rats

\begin{tabular}{|c|c|c|c|c|c|}
\hline \multirow[t]{2}{*}{ Group } & \multirow[t]{2}{*}{ Dose } & \multicolumn{2}{|l|}{$\mathrm{BW}(\mathrm{g})$} & \multirow{2}{*}{$\begin{array}{l}\text { Liver index } \\
\left(\mathrm{g} / 100 \mathrm{~g} \mathrm{BW}^{-1}\right)\end{array}$} & \multirow[t]{2}{*}{ Kidney inde } \\
\hline & & $\overline{\text { Initial }}$ & Final & & \\
\hline Control & & $241.25 \pm 6.50$ & $419.78 \pm 16.43$ & $3.47 \pm 0.07$ & $0.56 \pm 0.04$ \\
\hline Alcohol & & $237.26 \pm 4.59$ & $346.73 \pm 21.65^{* *}$ & $3.32 \pm 0.24$ & $0.53 \pm 0.01$ \\
\hline Silymarin & 100 mg/kg BW & $237.16 \pm 3.56$ & $315.40 \pm 24.93^{* * *}$ & $3.19 \pm 0.12$ & $0.55 \pm 0.03$ \\
\hline YGMM (low dose) & $1.0 \mathrm{ml} / \mathrm{kg} \mathrm{BW}$ & $238.16 \pm 3.46$ & $310.88 \pm 11.33^{* * *}$ & $3.71 \pm 0.11$ & $0.57 \pm 0.03$ \\
\hline YGMM (medium dose) & $2.5 \mathrm{ml} / \mathrm{kg} \mathrm{BW}$ & $239.54 \pm 4.24$ & $335.73 \pm 15.89^{* *}$ & $3.50 \pm 0.15$ & $0.60 \pm 0.04$ \\
\hline YGMM (high dose) & $5.0 \mathrm{ml} / \mathrm{kg} \mathrm{BW}$ & $234.26 \pm 4.12$ & $331.80 \pm 16.51^{* *}$ & $3.19 \pm 0.08$ & $0.61 \pm 0.02$ \\
\hline
\end{tabular}

Data are presented as mean \pm SEM in each group. Statistical significance is indicated by asterisks. ${ }^{* *} p<0.01,{ }^{* * *} p<0.001$ compared with normal control group. No significant differences were found between the alcohol group and other alcohol-treated groups

\section{Statistical analysis}

The results were reported as mean \pm SEM. The data were analyzed using IBM SPSS Statistics 20. Statistically significant data were further analyzed and compared using Duncan's multiple range test. Significant difference was considered at $p<0.05$.

\section{Results}

\section{General observation}

When treated with $14 \mathrm{ml} / \mathrm{kg}$ alcoholic liquor, the rats in every group (except the normal control) behaved excitedly, such as unsteady walking and accelerated breathing. Food utilization simultaneously began to decrease. Except for the normal group, the other groups did not exhibit mortality until the 15th day after alcohol administration. Average weight dramatically declined in the groups treated with alcohol (Table 1). The result shows that Red Star Erguotou Liquor significantly reduced the BW of rats. No significant differences were found between the alcoholic liquor group and other treated alcoholic liquor groups. As shown in Table 1, liver and kidney weight to BW ratios were similar, and no significant change was observed among the different groups.

\section{Effect of YGMM on ALT and AST levels}

As shown in Table 2, serum levels of AST and ALT in the alcohol group were significantly elevated after treatment with alcohol $(p<0.05)$, indicating the establishment of an acute alcohol-induced liver injury model in rats. YGMM exhibited a curative effect on the acute alcohol-induced liver injury by dose-dependently reducing the AST and ALT levels in rats. Finally, the AST and ALT levels of YGMM group (dose of $5 \mathrm{ml} / \mathrm{kg}$ ) decreased by 40.86 and $24.71 \%$, respectively, as compared with those in the alcohol group.

\section{Effect of YGMM on TB and TG levels}

TB and TG contents in serum are presented in Table 2. TB and TG levels in the alcohol group were significantly increased. The administration of YGMM remarkably prevented the elevation of TB level in a dose-dependent manner. However, TG level did not show the same trend. No significant differences in TG level were found among YGMM groups, indicating that the effect of YGMM on TG level was not related to the dose within a certain concentration range.

\section{Effect of YGMM on T-SOD, GSH, and MDA levels}

The T-SOD, GSH, and MDA levels in the liver and kidney are shown in Table 3. Compared with that in the control group, the GSH level in the alcohol group was significantly decreased $(p<0.05)$. However, T-SOD and MDA levels showed no significant change. The treatment of YGMM slightly recovered the decreased T-SOD and GSH levels. This obscure effect resulted from the mild oxidative damage in this study.

Table 2 Effect of YGMM on ALT, AST, TG and TB levels in control and alcohol-treated rats

\begin{tabular}{llllll}
\hline Group & ALT (U/l) & AST (U/l) & TG (mg/dl) & TB (mg/dl) & AST/ALT \\
\hline Control & $8.49 \pm 0.33$ & $21.70 \pm 1.10$ & $3.75 \pm 0.56$ & $0.28 \pm 0.02$ & $2.57 \pm 0.15$ \\
Alcohol & $11.37 \pm 0.37^{\# \#}$ & $31.28 \pm 1.95^{\# \# \#}$ & $10.50 \pm 2.14^{\# \#}$ & $0.47 \pm 0.04^{\# \# \#}$ & $2.77 \pm 0.23$ \\
Silymarin & $8.09 \pm 0.47^{* *}$ & $24.16 \pm 3.56^{*}$ & $2.67 \pm 0.61^{* * *}$ & $0.32 \pm 0.03^{* *}$ & $3.03 \pm 0.55$ \\
YGMM (low dose) & $9.35 \pm 1.12$ & $22.24 \pm 2.23^{* *}$ & $3.08 \pm 1.09^{* *}$ & $0.33 \pm 0.03^{* *}$ & $2.41 \pm 0.13$ \\
YGMM (medium dose) & $8.34 \pm 1.44^{*}$ & $19.79 \pm 0.58^{* * *}$ & $4.77 \pm 1.42^{*}$ & $0.26 \pm 0.06^{* * *}$ & $2.52 \pm 0.43$ \\
YGMM (high dose) & $8.56 \pm 0.41^{*}$ & $18.50 \pm 1.88^{* * *}$ & $4.89 \pm 1.80^{*}$ & $0.26 \pm 0.01^{* * *}$ & $2.15 \pm 0.14$ \\
\hline
\end{tabular}

Data are presented as mean \pm SEM in each group. Statistically significant differences are indicated by asterisks and pound sign $\left({ }^{*} p<0.05,{ }^{* *} p<0.01,{ }^{* * *} p<0.001\right.$

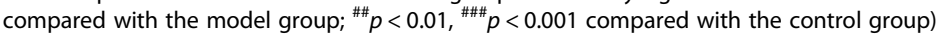


Table 3 Effect of YGMM on T-SOD, GSH, and MDA levels in alcoholic liver injury of rats

\begin{tabular}{|c|c|c|c|c|c|c|}
\hline \multirow[t]{2}{*}{ Group } & \multicolumn{3}{|l|}{ Liver } & \multicolumn{3}{|l|}{ Kidney } \\
\hline & $\begin{array}{l}\text { T-SOD } \\
\text { (U/mg protein) }\end{array}$ & $\begin{array}{l}\text { GSH } \\
\text { (mg/g protein) }\end{array}$ & $\begin{array}{l}\text { MDA } \\
\text { (nmol/mg protein) }\end{array}$ & $\begin{array}{l}\text { T-SOD } \\
\text { (U/mg protein) }\end{array}$ & $\begin{array}{l}\text { GSH } \\
\text { (mg/g protein) }\end{array}$ & $\begin{array}{l}\text { MDA } \\
\text { (nmol/mg protein) }\end{array}$ \\
\hline Control & $214.67 \pm 22.34$ & $3.32 \pm 0.37$ & $1.53 \pm 0.20$ & $127.90 \pm 15.81$ & $3.95 \pm 0.19$ & $0.88 \pm 0.04$ \\
\hline Alcohol & $180.71 \pm 5.71$ & $2.43 \pm 0.24^{\#}$ & $1.57 \pm 0.11$ & $99.43 \pm 4.17$ & $3.39 \pm 0.11^{\#}$ & $0.77 \pm 0.04$ \\
\hline Silymarin & $199.52 \pm 6.48$ & $2.91 \pm 0.11$ & $1.57 \pm 0.16$ & $86.15 \pm 3.30$ & $3.03 \pm 0.14$ & $1.22 \pm 0.07^{* * *}$ \\
\hline YGMM (low dose) & $220.10 \pm 18.50$ & $3.18 \pm 0.23^{*}$ & $1.61 \pm 0.13$ & $100.47 \pm 14.39$ & $3.72 \pm 0.16$ & $0.86 \pm 0.06$ \\
\hline YGMM (medium dose) & $209.49 \pm 8.93$ & $2.86 \pm 0.22$ & $1.70 \pm 0.24$ & $85.07 \pm 3.31$ & $3.05 \pm 0.19$ & $0.73 \pm 0.08$ \\
\hline YGMM (high dose) & $210.86 \pm 19.61$ & $2.96 \pm 0.03$ & $1.83 \pm 0.13$ & $96.94 \pm 4.12$ & $3.42 \pm 0.15$ & $0.47 \pm 0.10^{* *}$ \\
\hline
\end{tabular}

Data are presented as mean \pm SEM in each group. ${ }^{*} p<0.05$ compared with the normal control group; ${ }^{*} p<0.05,{ }^{* * *} p<0.01,{ }^{* * *} p<0.001$ compared with the alcohol group

\section{Histopathological studies}

The livers of the normal control group exhibited resilience in a bright color after being separated from the rat body; however, those in the alcohol group were dim and swelling. The histological features are shown in Fig. 2. In the normal control group, the liver lobular architecture was clear, the central veins were intact, the hepatic cell cords were arranged neatly, and the cells were uniform in size. No pathological changes were detected in the normal control. However, the alcohol group showed some liver pathological changes, which are characterized by the cellular edema, inflammatory cell infiltrates. Histopathological changes caused by alcohol were improved by YGMM. The best therapeutic effect was achieved at a dose of $5 \mathrm{ml} /$
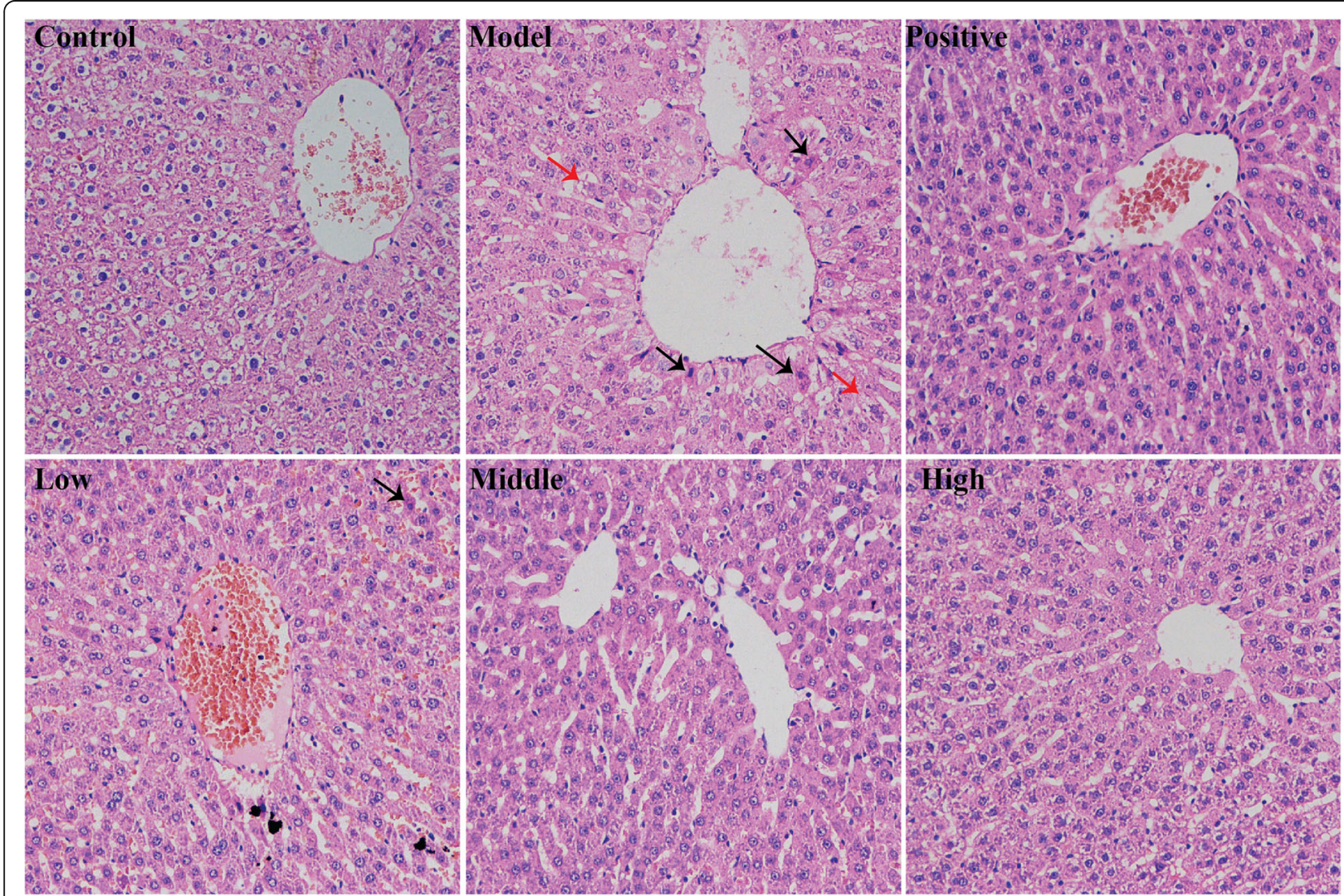

Fig. 2 Light microscopic analysis of rat liver sections of normal rats and alcohol treatment with or without YGMM administration (H\&E staining, 200x). Control group was given equal volumes of physiological saline, model group was treated with alcohol, positive group was treated with alcohol and Silymarin $(100 \mathrm{mg} / \mathrm{kg})$, low group was treated with alcohol and YGMM $(1 \mathrm{ml} / \mathrm{kg})$, middle group was treated with alcohol and YGMM $(2.5 \mathrm{ml} / \mathrm{kg})$, high group was treated with alcohol and YGMM $(5 \mathrm{ml} / \mathrm{kg})$. Black arrow showed inflammatory cells, and red arrow showed the swelling of liver cells 
$\mathrm{kg}$, which was similar to the silymarin group and was remarkable distinction with the alcohol group.

In the liver tissues and cells, Oil Red O can dissolve in lipid combined with triglyceride and become orange lipid droplets. After staining with Oil Red O, abundant orange lipid droplets were found around the central veins in the alcohol group (Fig. 3 a). These lipid droplets were sharply reduced in the silymarin and YGMM groups (Fig. 3b), indicating that YGMM had a positive therapeutic effect for alcoholic fatty liver.

\section{Discussion}

Many effective and accurate animal models have been developed to establish a liver pathology that is completely analogous to the clinical situation for ALD. Rodents and oral administration are the most suitable option for the animal model of ALD [39]. Wang et al. gave rats $50 \%$ alcohol at a dose of $12 \mathrm{ml} / \mathrm{kg}$ for 8 days [40], and another different method was that the mice orally received $2.4 \mathrm{~g} / \mathrm{kg}$ of ethanol for 15 days [41]. Other researchers gave rats $6 \mathrm{~g} / \mathrm{kg}$ of alcohol for 4 weeks [42]. In the preliminary assay, the serum levels of ALT, AST, and TG were all significantly increased after 4 weeks of alcohol treatment in rats. As a consequence, the experiment was terminated, and blood and liver and kidney tissues were collected on the 28th day of alcohol administration in our formal tests. Given the strong aversion of rats to alcohol, the consumption of animal feed was reduced after alcohol administration. In the present study, the BW of rats significantly decreased after alcohol treatment, which was not restored by YGMM (Table 1).

ALT and AST are important metabolic enzymes in liver cells and are usually at a low level in the plasma. When the structural integrity of hepatic cells and even organelles such as mitochondria were damaged from xenobiotics, soluble enzymes such as ALT and AST compartmented will be released into the blood [43]. Therefore, the serum transaminases (ALT and AST) usually are regarded as the optimum markers to diagnose liver injury [44]. In our study, significant increases of ALT and AST levels were obtained after administration with alcohol (Table 2), which indicated that alcohol treatment could damage the plasma and organelle membranes. YGMM pretreatment attenuated ALT and AST elevation in a dose-dependent manner (Table 2). The change complied with a universal viewpoint that the ALT and AST content recovered to a general level accompanied by liver parenchyma rehabilitation and hepatic cell regeneration [45].

Pyridoxal 5' -phosphate is an important ALT and AST coenzyme. Pyridoxal 5' - phosphate deficiency is common in ALD [46]. Its depletion causes a reduction of liver ALT at a certain degree than AST [47]. As a result, AST/ALT value is increased, which is regarded as typical in patients with alcoholic hepatitis [48]. This increased value in alcohol-related hepatic disease was found in 1967 [49]. The same changing trend could be found in
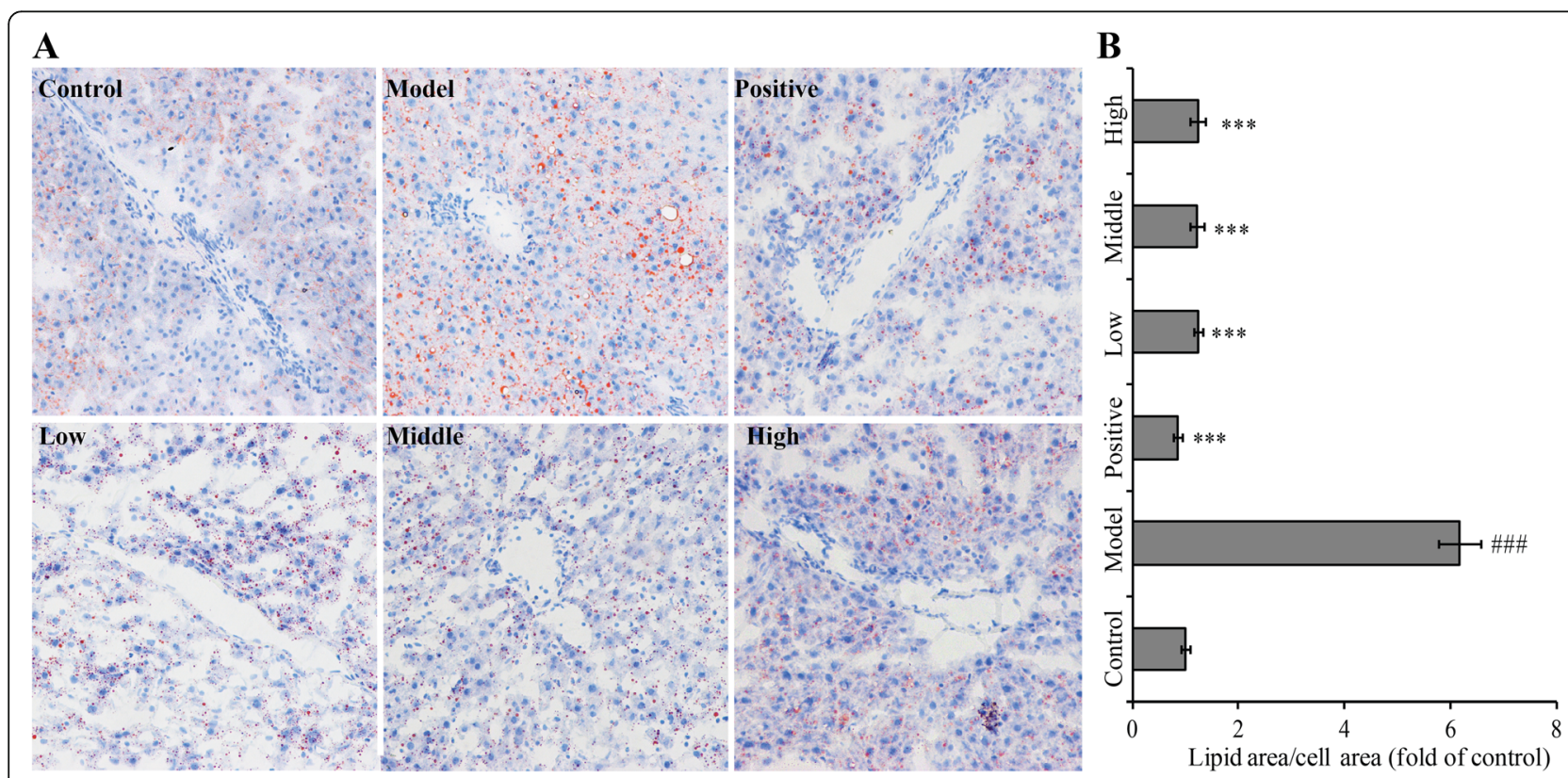

Fig. 3 Oil Red O staining for qualitatively and quantitatively visualizing hepatic lipid accumulation under light microscope (200x). Control group was given equal volumes of physiological saline, model group was treated with alcohol, positive group was treated with alcohol and Silymarin $(100 \mathrm{mg} / \mathrm{kg})$, low group was treated with alcohol and YGMM $(1 \mathrm{ml} / \mathrm{kg})$, middle group was treated with alcohol and YGMM $(2.5 \mathrm{ml} / \mathrm{kg})$, high group was treated with alcohol and YGMM $(5 \mathrm{ml} / \mathrm{kg}){ }^{\# \# \#} p<0.001$ compared with the control group, ${ }^{* * *} p<0.001$ compared with the model group 
people suffering from alcoholic hepatitis and acute liver injury in other studies $[50,51]$. In the present study, the ratio of AST/ALT was increased in the alcohol group compared with normal control, which indicated an advanced ALD, although no significant change was observed (Table 2). A similar result was also reported by Lin et al. [42]. YGMM treatment inhibited the increase in AST/ALT ratio (Table 2), which may alleviate alcoholic hepatic injury.

Bilirubin is a final product of heme degradation by heme oxygenase. Biliverdin, which is converted by heme oxygenase, is the intermediate degradation product of bilirubin $[52,53]$. Bilirubin is a potent antioxidant that can effectively remove ROS and nitric oxide $[54,55]$. Accumulating evidence has shown that serum total bilirubin concentration is independently and inversely associated with the progression of diabetic nephropathy, coronary atherosclerosis, and type 2 diabetes with retinopathy [56-58]. In contrast to the aforementioned disorders, high serum total bilirubin concentration was observed in ALD and non-ALD patients [59, 60]. A possible mechanism for the increase in alcohol consumption is that alcohol competitively inhibits bilirubin conjugation [60]. UDP-glucuronosyltransferase (UGT1A1), a metabolizing enzyme preventing bilirubin accumulation to toxic levels [61], is involved in the pathway for glucuronidation of alcohol and bilirubin. Hence, the presence of alcohol will affect bilirubin conjugation by a competitive relationship. Although an elevation of UGT1A1 mRNA and microsomal UGT1A1 protein was observed in the liver of ethanol consumer animals [62], the compensation is not effective in the condition with high concentrations of alcohol substrate. In the current study, the alcohol group had a significant increase $(167.86 \%)$ of serum total bilirubin level compared with the normal control group. However, YGMM treatment obviously recovered the serum total bilirubin content (Table 2). This therapeutic effect of YGMM may be due to stimulating the pathway for glucuronidation of bilirubin.

Studies show that fat accumulation is universal in hepatic cells treated with alcohol $[63,64]$. Fat accumulation in ALD is a complicated process. The exact mechanisms underlying steatohepatitis, including fatty acid synthesis and oxidation, are primarily regulated by SREBP- 1 and PPAR- $\alpha$ [65]. Alcohol inhibits PPAR- $\alpha$ transcriptional activating and DNA combining capacity, which is then influenced by dissociative fatty acid transportation and oxidization. Alcohol exposure also elevates SREBP-1 content and promotes the synthesis of fatty acids [65]. In the present study, the serum level of TG contents in the alcohol group was increased by $280 \%$ compared with that in the normal control group; however, YGMM treatment significantly reduced the serum TG level (Table 2). Oil Red O staining further confirmed the increase in lipid level (Fig. 3b). YGMM treatment (doses of 2.5 and $5 \mathrm{ml} / \mathrm{kg}$ ) recovered the pathologic lesions similar to silymarin. The increase in serum TG level and lipid change in hepatocytes indicated that alcoholic fatty liver was already established in the animal model. In this study, YGMM treatment could cease fat accumulation caused by alcohol exposure. This therapeutic effect of YGMM may be facilitated through the above molecular pathways. Another possible pathway is the altered $\mathrm{NADH} / \mathrm{NAD}^{+}$redox potential, which has long been regarded as the way that alcohol causes fatty liver [65]. In this pathway, alcohol-induced fatty liver could be prevented by antioxidants [66]. Therefore, the effect of YGMM on serum TG level may explain clearly with the antioxidants contained in YGMM. Substantial alcohol may become the preferential fuel in hepatic cells and turn it into important energy source instead of fat, which supports fatty acid accumulation [67]. Hyperlipidemia prevails in a minimal liver damage at the beginning of alcohol consumption but has an opposite trend in a severe liver injury.

Oxidative stress, which is involved in alcohol abuse, can damage antioxidant defenses and produce ROS at the same time [68]. T-SOD, GSH, and MDA contents were measured in the liver and kidney tissues of each group to detect the oxidative damage induced by alcohol in the current study. Alcohol treatment exhibited 1.37fold less GSH in liver tissues and 1.17-fold less GSH in kidney tissues compared with the control group. By contrast, the value of T-SOD and MDA showed no significant change. These data showed that mild oxidative damage was present in these two organs in the current assay. Thus, the hepatoprotective activity of YGMM for oxidative injury can be disregarded. In a previous study, a generous alcohol dose did not induce oxidative stress in livers of male SD rats [69]. Alcohol inhibited GSH synthesis and increased the consumption of GSH due to oxidation [70]. As a result, hepatic and nephritic GSH levels were decreased significantly in the current study. GSH is a key intracellular antioxidant [67], and increased consumption of GSH helps to keep a stabilized intracellular environment against pro-oxidants and antioxidants. Therefore, mild oxidative damage was obtained in this study. Alcohol-induced oxidative damage is considered to be a crucial player in the mechanisms by which alcohol produces liver injury. Cytochrome P450 $2 \mathrm{E} 1$ is a critical enzyme in this process, which generates $\mathrm{O}_{2}{ }^{--}$and $\mathrm{H}_{2} \mathrm{O}_{2}$ [70]. $\mathrm{O}_{2}{ }^{\cdot-}$ can be transformed to $\mathrm{H}_{2} \mathrm{O}_{2}$ by T-SOD, and then catalase transforms $\mathrm{H}_{2} \mathrm{O}_{2}$ to $\mathrm{H}_{2} \mathrm{O}$. Another widely accepted idea, where MDA is closely related with cytochrome content, can be taken into consideration [71]. In this case, when oxidative damage was mild due to the increased consumption of GSH, the content of T-SOD and MDA ranged in the same level after 
alcohol treatment. No significant change in SOD level after alcohol administration $(5 \mathrm{~g} / \mathrm{kg})$ to rats was observed by Develi et al. [72].

Yigan mingmu in Chinese means "benefits the liver and brightens the eyes." To explore the hepatoprotective effect of YGMM, we established an acute alcohol-induced liver injury model. Our data revealed that YGMM treatment decreased significantly the high levels of ALT, AST, TB, and TG caused by alcohol exposure. Even so, the current work was the preliminary experimental study of YGMM under the ideal conditions, in which had the appropriate alcohol dose to induce the liver injury and proper volume of YGMM to prevent the progress of ALD. A better understand of YGMM to decrease fat accumulation needs to consider the human alcohol intake and dosage range of YGMM in daily life, and clinical experiments with more sample size. Meanwhile, further studies on the chemical composition analysis and molecular mechanism of hepatoprotective activity are necessary to understand the vital role of YGMM against acute alcohol-induced liver injury.

\section{Conclusion}

YGMM exhibits an attractive protective effect against acute liver injury induced by alcohol in rats. The decreased ALT, AST, and TG levels indicated that YGMM could restore the damage in hepatic cell and cease fat accumulation. These properties are considered as the primary mechanisms of YGMM to prevent the progress of ALD. This study presented persuasive results to support the hepatoprotective activity of YGMM.

\section{Abbreviations}

ALD: Alcoholic liver disease; ALT: Alanine transferase; AST: Aspartate transaminase; GSH: Glutathione; MDA: Malondialdehyde; PPAR-a: peroxisome proliferator-activated receptor-a; SREBP-1: sterol regulatory element binding protein 1; TB: Total bilirubin; TCM: Traditional Chinese Medicine;

TG: Triglyceride; T-SOD: Total superoxide dismutase

\section{Acknowledgments}

No applicable.

\begin{abstract}
Authors' contributions
Conceived and designed the experiments: YWW, JHZ; Prepared and analyzed the YGMM: BBX, XYZ, LYC; Performed the experiments: MQG, ZG, MBX, CYX; Analyzed the data: MQG, YWW; Wrote the paper: MQG, YWW; Revision and feedback of article done: YWW, JHZ. All authors read and approved the final manuscript
\end{abstract}

\section{Funding}

No applicable.

\section{Availability of data and materials}

All data generated or analysed during the current study are included in this article.

\section{Ethics approval and consent to participate}

The experimental protocol was approved by the Animal Ethics Committee of Wuhan University, Wuhan, China. Animal assays were conducted according to the guidelines of the Committee for the Purpose of Control and Supervision of Experiments on Animals.
Consent for publication

No applicable.

\section{Competing interests}

The authors declare that they have no competing interests.

\section{Author details}

${ }^{1}$ Institute of TCM and Natural Products, School of Pharmaceutical Sciences, Wuhan University, Wuhan 430071, People's Republic of China. ${ }^{2}$ Beijing Hebabiz Biotechnology Co. Ltd, Beijing 102206, People's Republic of China. ${ }^{3}$ Guangxi Hebabiz Pharmaceutical Co. Ltd, National and Region joint Engineering Center for Anticancer Drug Development, Qinzhou 535008, People's Republic of China. ${ }^{4}$ MOE Key Laboratory of Combinatorial Biosynthesis and Drug Discovery, Wuhan University, Wuhan 430072, People's Republic of China.

Received: 2 November 2017 Accepted: 15 January 2020

Published online: 05 February 2020

\section{References}

1. Seitz HK, Bataller R, Cortez-Pinto H, Gao B, Gual A, Lackner C, Mathurin P, Mueller S, Szabo G, Tsukamoto H. Alcoholic liver disease. Nat Rev Dis Primers. 2018;4(1):1-22.

2. Lin CW, Zhang H, Li M, Xiong X, Chen X, Chen X, Dong XC, Yin XM. Pharmacological promotion of autophagy alleviates steatosis and injury in alcoholic and non-alcoholic fatty liver conditions in mice. J Hepatol. 2013; 58(5):993-9.

3. Goncalves JL, Queiroz NL, Sabino JFL, Marques PE, Galvao I, Gamba CO, Cassali GD, Carvalho LM, Silva DAS, Versiani A, Teixeira MM, Faria AMC, Vieira AT, Godard ALB. Evaluating the effects of refined carbohydrate and fat diets with acute ethanol consumption using a mouse model of alcoholic liver injury. J Nutr Biochem. 2017:39:93-100.

4. Kwong EK, Liu RP, Zhao D, Li XJ, Zhu WW, Wang X, Gurley EC, Lai GH, Liu JM, Hylemon PB, Zhou HP. The role of sphingosine kinase 2 in alcoholic liver disease. Dig Liver Dis. 2019;51(8):1154-63.

5. Stewart S, Jones D, P. Day C. Alcoholic liver disease: new insights into mechanisms and preventative strategies. Trends Mol Med. 2001;7(9):408-13.

6. Wang M, Zhu P, Jiang C, Ma L, Zhang Z, Zeng X. Preliminary characterization, antioxidant activity in vitro and hepatoprotective effect on acute alcohol-induced liver injury in mice of polysaccharides from the peduncles of Hovenia dulcis. Food Chem Toxicol. 2012;50(9):2964-70.

7. Bouneva I, Abou-Assi S, Heuman DM, Mihas AA. Alcoholic liver disease. Hosp Physician. 2003;39(10):31-8.

8. Zhao J, Chen H, Li Y. Protective effect of bicyclol on acute alcohol-induced liver injury in mice. Eur J Pharmacol. 2008;586(1-3):322-31.

9. Gao HY, Huang J, Wang HY, Du XW, Cheng SM, Han Y, Wang LF, Li GY, Wang JH. Protective effect of Zhuyeqing liquor, a Chinese traditional health liquor, on acute alcohol-induced liver injury in mice. Int J Inflamm. 2013; 10(1):30-7.

10. Kim SJ, Lee SM. Effect of baicalin on toll-like receptor 4-mediated ischemia/ reperfusion inflammatory responses in alcoholic fatty liver condition. Toxicol Appl Pharmacol. 2012;258(1):43-50.

11. Ding RB, Tian $K$, Huang LL, He CW, Jiang Y, Wang YT, Wan JB. Herbal medicines for the prevention of alcoholic liver disease: a review. J Ethnopharmacol. 2012;144(3):457-65.

12. Zeng T, Zhang CL, Zhao N, Guan MJ, Xiao M, Yang R, Zhao XL, Yu LH, Zhu ZP, Xie KQ. Impairment of Akt activity by CYP2E1 mediated oxidative stress is involved in chronic ethanol-induced fatty liver. Redox Biol. 2018;14:295-304.

13. Song XL, Shen $Q$, Liu M, Zhang C, Zhang L, Ren ZZ, Wang WS, Dong YH, Wang XX, Zhang JJ, Jia L. Antioxidant and hepatoprotective effects of intracellular mycelium polysaccharides from Pleurotus geesteranus against alcoholic liver diseases. Int J Biol Macromol. 2018;114:979-88.

14. Yang KT, Lin YL, Lin YX, Wang SY, Wu YHS, Chou CH, Fu SG, Chen YC. Protective effects of antioxidant egg-chalaza hydrolysates against chronic alcohol consumption-induced liver steatosis in mice. J Sci Food Agric. 2019; 99(5):2300-10

15. Zhu H, Jia ZQ, Misra H, Li YR. Oxidative stress and redox signaling mechanisms of alcoholic liver disease: updated experimental and clinical evidence. J Dig Dis. 2012;13(3):133-42. 
16. Xiong SL, Li AL, Huang N, Lu F, Hou DB. Antioxidant and immunoregulatory activity of different polysaccharide fractions from tuber of Ophiopogon japonicus. Carbohydr Polym. 2011;86(3):1273-80.

17. Li HT, Wu M, Jiang J, Sun XM, Chen LJ, Feng M, Yuan DY, Wen ZY, Qin CJ. The extracts of Angelica sinensis restore the digestive and absorptive capacity through improving antioxidant status in digestive organs of fish treated with trichlorfon. Aquacult Res. 2019;50(2):490-504

18. Bae SM, Na AS, Seo HK, Lee SC. Effects of drying conditions on the antioxidant activities and volatile compounds of Chrysanthemi Flos flowers. J Food Sci Nutr. 2009;14(4):329-34.

19. Liu CJ, Liu Q, Sun JD, Jiang B, Yang JF. Extraction of water-soluble polysaccharide and the antioxidant activity from Semen cassiae. J Food Drug Anal. 2014;22(4):492-9.

20. Fu MQ, An KJ, Xu YJ, Chen YL, Wu JJ, Yu YS, Zou B, Xiao GS, Ti H. Effects of different temperature and humidity on bioactive flavonoids and antioxidant activity in Pericarpium Citri Reticulata (Citrus reticulata 'Chachi'). LWT-Food Sci Technol. 2018;93:167-73.

21. Hu J, Jia XJ, Fang XB, Li P, He CW, Chen MW. Ultrasonic extraction, antioxidant and anticancer activities of novel novelpolysaccharides from Chuanxiong rhizome. Int J Biol Macromol. 2016;85:277-84.

22. Seo MK, Cho HY, Lee CH, Koo KA, Park YK, Lee JG, Lee BJ, Park SW, Kim YH. Antioxidant and proliferative activities of Bupleuri Radix extract against serum deprivation in SH-SY5Y cells. Psychiatry Investig. 2013;10(1):81-8.

23. Waisundara $V Y$, Huang MQ, Hsu A, Huang DJ, Tan BKH. Characterization of the anti-diabetic and antioxidant effects of Rehmannia glutinosa in streptozotocininduced diabetic Wistar rats. Am J Chin Med. 2008;36(06):1083-104.

24. Chotimarkorn $\mathrm{C}$, Ushio $\mathrm{H}$. The effect of trans-ferulic acid and gammaoryzanol on ethanol-induced liver injury in C57BL mouse. Phytomedicine. 2008;15(11):951-8

25. Wang LY, Tang YP, Liu X, Zhu M, Tao WW, Li WX, Duan JA. Effects of ferulic acid on antioxidant activity in Angelicae Sinensis Radix, Chuanxiong Rhizoma, and their combination. Chin J Nat Med. 2015;13(6):401-8.

26. He DY, Dai SM. Anti-inflammatory and immunomodulatory effects of Paeonia lactiflora Pall., a traditional chinese herbal medicine. Front Pharmacol. 2011;2:10.

27. Le K, Chiu F, Ng K. Identification and quantification of antioxidants in Fructus lycii. Food Chem. 2007;105(1):353-63.

28. Oh HL, Ahn MH, Kim NY, Song JE. Quality characteristics and antioxidant activities of Yanggeng with added Rehmanniae Radix preparata concentrate. Korean J Food Cookery Sci. 2012;28(1):1-8.

29. Matsuda H, Murakami T, Ninomiya K, Inadzuki M, Yoshikawa M. New hepatoprotective saponins, bupleurosides III, VI, IX and VII, from Chinese Bupleuri radix: structure-requirements for the cytoprotective activity in primary cultured rat hepatocytes. Bioorg Med Chem Lett. 1997;7(17):2193-8.

30. Choo MH, Jeong $Y$, Lee MY. Effects of an ethylacetate fraction of Chrysanthemi Flos on the antioxidative system and lipid profile in rats with ethanol-induced liver damage. J Food Sci Nutr. 2004;9(4):352-60.

31. Lieber CS, Rubin E. Alcoholic fatty liver in man on a high protein and low fat diet. Am J Med. 1968:44(2):200-6.

32. Lieber CS. Alcoholic fatty liver: its pathogenesis and mechanism of progression to inflammation and fibrosis. Alcohol. 2004;34(1):9-19.

33. Xu AM, Wang Y, Keshaw H, Xu LY, Lam KSL, Gooper GJS. The fat-derived hormone adiponectin alleviates alcoholic and nonalcoholic fatty liver diseases in mice. J Clin Invest. 2003;112(1):91-100.

34. Tzeng TF, Lu HJ, Liou SS, Chang CJ, Liu IM. Vinegar-baked Radix Bupleuri regulates lipid disorders via a pathway dependent on peroxisomeproliferator -activated receptor- $a$ in high-fat-diet-induced obese rats. Evid Based Complement Altern. 2012;2012:827278.

35. Gu M, Zhang Y, Fan SJ, Ding XB, Ji G, Huang C. Extracts of Rhizoma Polygonati odorati prevent high-fat diet-induced metabolic disorders in C57BL/6 mice. PLoS One. 2013;8(11):e81724

36. Hu SL, Shen G, Zhao WG, Wang F, Jiang XD, Huang DB. Paeonol, the main active principles of Paeonia moutan, ameliorates alcoholic steatohepatitis in mice. J Ethnopharmacol. 2010;128(1):100-6.

37. Wang Y, Zhu YY, Ruan KF, Wei H. MDG-1, a polysaccharide from Ophiopogon japonicus, prevents high fat diet-induced obesity and increases energy expenditure in mice. Carbohydr Polym. 2014;114:183-9.

38. Xu WX, Lu CF, Yao L, Zhang F, Shao JJ, Zheng SZ. Dihydroartemisinin protects against alcoholic liver injury through alleviating hepatocyte steatosis in a farnesoid $X$ receptor-dependent manner. Toxicol Appl Pharmacol. 2017;315:23-34.
39. Arteel GE. Animal models of alcoholic liver disease. Dig Dis. 2010;28(6):729-36.

40. Wang H, Feng F, Zhuang BY, Sun Y. Evaluation of hepatoprotective effect of Zhi-Zi-Da-Huang decoction and its two fractions against acute alcoholinduced liver injury in rats. J Ethnopharmacol. 2009;126(2):273-9.

41. Wang XY, Luo JP, Chen R, Zha XQ, Pan LH. Dendrobium huoshanense polysaccharide prevents ethanol-induced liver injury in mice by metabolomic analysis. Int J Biol Macromol. 2015;78:354-62.

42. Lin B, Zhang F, Yu Y, Jiang Q, Zhang Z, Wang J, Li Y. Marine collagen peptides protect against early alcoholic liver injury in rats. Brit J Nutr. 2012; 107(8):1160-6.

43. Rajagopal SK, Manickam P, Periyasamy V, Namasivayam N. Activity of Cassia auriculata leaf extract in rats with alcoholic liver injury. J Nutr Biochem. 2003:14(8):452-8.

44. Chenoweth $\mathrm{MB}$, Hake $\mathrm{CL}$. The smaller halogenated aliphatic hydrocarbons. Annu Rev Pharmacol. 1962;2(1):363-98.

45. Thabrew Ml, Joice PD, Rajatissa W. A comparative study of the efficacy of Pavetta indica and Osbeckia octandra in the treatment of liver dysfunction. Planta Med. 1987;53(03):239-41.

46. Lumeng L, Li TK. Vitamin $B_{6}$ metabolism in chronic alcohol abuse. Pyridoxal phosphate levels in plasma and the effects of acetaldehyde on pyridoxal phosphate synthesis and degredation in human erythrocytes. J Clin Invest. 1974;53(3):693-704.

47. Das SK, Nayak P, Vasudevan DM. Biochemical markers of alcohol consumption. Indian J Clin Biochem. 2003;18(2):111-8.

48. Diehl AM, Potter J, Boitnott J, Van Duyn MA, Herlong HF, Mezey E. Relationship between pyridoxal 5'-phosphate deficiency and aminotransferase levels in alcoholic hepatitis. Gastroenterology. 1984;86(4):632-6.

49. Harinasuta U, Chome TB, Jshak K, Zimmerman HJ. Steatonecrosis-Mallory body type. Medicine. 1967;64(2):141-62.

50. Das SK, Vasudevan DM. Biochemical diagnosis of alcoholism. Indian J Clin Biochem. 2005;20(1):35-42.

51. Nyblom H, Berggren U, Balldin J, Olsson R. High AST/ALT ratio may indicate advanced alcoholic liver disease rather than heavy drinking. Alcohol Alcohol. 2004;39(4):336-9.

52. Tell G, Gustincich S. Redox state, oxidative stress, and molecular mechanisms of protective and toxic effects of bilirubin on cells. Curr Pharm design. 2009;15(25):2908-14.

53. Vitek L, Ostrow JD. Bilirubin chemistry and metabolism; harmful and protective aspects. Curr Pharm Design. 2009;15(25):2869-83.

54. Mancuso C, Pani G, Calabrese V. Bilirubin: an endogenous scavenger of nitric oxide and reactive nitrogen species. Redox Rep. 2006;11(5):207-13.

55. Macías-García D, Méndez-Del Barrio C, Jesús S, Labrador MA, Adarmes-Gómez A, Vargas-González L, Carrillo F, Gómez-Garre P, Mir P. Increased bilirubin levels in Parkinson's disease. Parkinsonism Relat Disord. 2019;63:213-6.

56. Akboga MK, Canpolat U, Sahinarslan A, Alsancak Y, Nurkoc S, Aras D, Aydogdu S, Abaci A. Association of serum total bilirubin level with severity of coronary atherosclerosis is linked to systemic inflammation. Atherosclerosis. 2015;240(1):110-4.

57. Sakoh T, Nakayama M, Tanaka S, Yoshitomi $R$, Ura Y, Nishimoto H, Fukui A, Shikuwa Y, Tsuruya K, Kitazono T. Association of serum total bilirubin with renal outcome in Japanese patients with stages 3-5 chronic kidney disease. Metabolism. 2015;64(9):1096-102.

58. Sekioka R, Tanaka M, Nishimura T, Itoh H. Serum total bilirubin concentration is negatively associated with increasing severity of retinopathy in patients with type 2 diabetes mellitus. J Diabetes Complications. 2015;29(2):218-21.

59. Ahlgren A, Hedenborg G, Norman A, Wisen O. Serum bilirubin subfractions in patients with alcohol abuse during detoxication. Scand J Clin Lab Invest. 1988:48(4):319-26.

60. O'Malley SS, Gueorguieva R, Wu R, Jatlow PI. Acute alcohol consumption elevates serum bilirubin: an endogenous antioxidant. Drug Alcohol Depend. 2015;149:87-92

61. Lv X, Ge GB, Feng L, Troberg J, Hu LH, Hou J, Cheng HL, Wang P, Liu ZM, Finel $M$, Cui JN, Yang L. An optimized ratiometric fluorescent probe for sensing human UDP-glucuronosyltransferase $1 \mathrm{~A} 1$ and its biological applications. Biosens Bioelectron. 2015;72:261-7.

62. Kardon T, Coffey MJ, Banhegyi G, Conley AA, Burchell B, Mandl J, Braun L. Transcriptional induction of bilirubin UDP-glucuronosyltransferase by ethanol in rat liver. Alcohol. 2000;21(3):251-7.

63. Barson JR, Karatayev O, Chang GQ, Johnson DF, Bocarsly ME, Hoebel BG, Leibowitz SF. Positive relationship between dietary fat, ethanol intake, 
triglycerides, and hypothalamic peptides: counteraction by lipid-lowering drugs. Alcohol. 2009;43(6):433-41.

64. Yuan Q, Hou SS, Zhai JF, Tian T, Wu YJ, Wu ZL, He JS, Chen ZN, Zhang J. S100A4 promotes inflammation but suppresses lipid accumulation via the STAT3 pathway in chronic ethanol-induced fatty liver. J Mol Med. 2019; 97(4):1-14.

65. You M, Crabb DW. Recent advances in alcoholic liver disease II. Minireview: molecular mechanisms of alcoholic fatty liver. Am J Physiol Gastrointest Liver Physiol. 2004;287(1):G1-6.

66. Cederbaum Al. Introduction: role of lipid peroxidation and oxidative stress in alcohol toxicity. Free Radical Bio Med. 1989;7(5):537-9.

67. Das SK, Vasudevan DM. Alcohol-induced oxidative stress. Life Sci. 2007;81(3): $177-87$

68. Albano E. Alcohol, oxidative stress and free radical damage. P Nutr Soc. 2007:65(3):278-90

69. Lauterberg BH, Davies S, Mitchell JR. Ethanol suppresses hepatic glutathione synthesis in rats in vivo. J Pharmacol Exp Ther. 1984;230(1):7-11.

70. Cederbaum Al, Lu Y, Wu D. Role of oxidative stress in alcohol-induced liver injury. Arch Toxicol. 2009;83(6):519-48.

71. Zima T, Fialová L, Mestek O, Janebová M, Crkovská J, Malbohan I, Popov P. Oxidative stress, metabolism of ethanol and alcohol-related diseases. J Biomed Sci. 2001;8(1):59-70

72. Develi S, Evran B, Betül Kalaz E, Koçak-Toker N, Erata GÖ. Protective effect of Nigella sativa oil against binge ethanol-induced oxidative stress and liver injury in rats. Chin J Nat Med. 2014;12(7):495-9.

\section{Publisher's Note}

Springer Nature remains neutral with regard to jurisdictional claims in published maps and institutional affiliations.

Ready to submit your research? Choose BMC and benefit from:

- fast, convenient online submission

- thorough peer review by experienced researchers in your field

- rapid publication on acceptance

- support for research data, including large and complex data types

- gold Open Access which fosters wider collaboration and increased citations

- maximum visibility for your research: over $100 \mathrm{M}$ website views per year

At $\mathrm{BMC}$, research is always in progress.

Learn more biomedcentral.com/submissions 